\title{
Supporting insulin initiation in type 2 diabetes in primary care: results of the Stepping Up pragmatic cluster randomised controlled clinical trial
}

\author{
John Furler, ${ }^{1}$ David O’Neal, ${ }^{2}$ Jane Speight,, ${ }^{3,4,5}$ Jo-Anne Manski-Nankervis, ${ }^{1}$ Alexandra Gorelik, ${ }^{6}$ \\ Elizabeth Holmes-Truscott, ${ }^{3,4}$ Louise Ginnivan, ${ }^{3}$ Doris Young, ${ }^{1,7}$ James Best, ${ }^{8}$ Elizabeth Patterson, ${ }^{9}$ \\ Danny Liew, ${ }^{10}$ Leonie Segal, ${ }^{11}$ Carl May, ${ }^{12}$ Irene Blackberry ${ }^{13}$
}

\section{ABSTRACT}

OBJECTIVE

To compare the effectiveness of a novel model of care ("Stepping Up") with usual primary care in normalising insulin initiation for type 2 diabetes, leading to improved glycated haemoglobin (HbA1c) levels. DESIGN

Cluster randomised controlled trial.

SETTING

Primary care practices in Victoria, Australia, with a practice nurse and at least one consenting eligible patient ( $\mathrm{HbA} 1 \mathrm{C} \geq 7.5 \%$ with maximal oral treatment). PARTICIPANTS

266 patients with type 2 diabetes and 74 practices (mean cluster size 4 (range 1-8) patients), followed up for 12 months.

\section{INTERVENTION}

The Stepping Up model of care intervention involved theory based change in practice systems and reorientation of the roles of health professionals in the primary care diabetes team. The core components were an enhanced role for the practice nurse in leading insulin initiation and mentoring by a registered nurse with diabetes educator credentials.

MAIN OUTCOME MEASURES

The primary endpoint was change in $\mathrm{HbA1c}$. Secondary endpoints included the proportion of participants who

\section{WHAT IS ALREADY KNOWN ON THIS TOPIC}

Achieving and maintaining glycaemic targets early in type 2 diabetes improves long term outcomes

There are barriers to early stepwise progressive treatment intensification to achieve glycaemic targets in primary care, particularly when starting insulin

Interventions have had limited success in overcoming the delay in starting insulin treatment and changing clinical practice, in part because system level barriers are not addressed

\section{WHAT THIS STUDY ADDS}

Our model of care intervention changed clinical practice, with more patients in intervention arm practices starting insulin, with an overall benefit in terms of $\mathrm{HbA} 1 \mathrm{c}$ reduction, achieved without serious adverse events or any worsening in depressive symptoms

The theoretical base and flexible implementation are important characteristics of our intervention

Our model of care used existing resources and thus has important implications for policymakers, funders, and practitioners seeking innovative ways to provide the best care for people with type 2 diabetes in primary care transitioned to insulin, proportion who achieved target $\mathrm{HbA1c}$, and a change in depressive symptoms (patient health questionnaire, PHQ-9), diabetes specific distress (problem areas in diabetes scale, PAID), and generic health status (assessment of quality of life instrument, AQoL-8D).

\section{RESULTS}

$\mathrm{HbA1c}$ improved in both arms, with a clinically significant between arm difference (mean difference $-0.6 \%, 95 \%$ confidence interval $-0.9 \%$ to $-0.3 \%$ ), favouring the intervention. At 12 months, in intervention practices, 105/151 (70\%) of participants had started insulin, compared with 25/115 (22\%) in control practices (odds ratio $8.3,95 \%$ confidence interval 4.5 to $15.4, \mathrm{P}<0.001)$. Target $\mathrm{HbA} 1 \mathrm{c}(\leq 7 \%$ $(53 \mathrm{mmol} / \mathrm{mol}))$ was achieved by 54 (36\%) intervention participants and 22 (19\%) control participants (odds ratio 2.2, 1.2 to $4.3, \mathrm{P}=0.02$ ). Depressive symptoms did not worsen at 12 months (PHQ-9: -1.1 (3.5) $v-0.1$ (2.9), $\mathrm{P}=0.05)$. A statistically significant difference was found between arms in the mean change in mental health (AQoL mental component summary: 0.04 (SD 0.16) $v-0.002$ (0.13), mean difference 0.04 (95\% confidence interval 0.002 to 0.08$), P=0.04)$, favouring the intervention, but no significant difference in physical health (AQoL physical component summary: $0.03(0.15) v$ $0.02(0.13))$ nor diabetes specific distress (5.6 (15.5) $v-2.4$ (15.4)). No severe hypoglycaemia events were reported.

\section{CONCLUSIONS}

The Stepping Up model of care was associated with increased insulin initiation rates in primary care, and improvements in glycated haemoglobin without worsening emotional wellbeing.

\section{TRIAL REGISTRATION}

Australian and New Zealand Clinical Trials Registry ACTRN12612001028897.

\section{Introduction}

Nearly 600 million people worldwide will have type 2 diabetes by $2030 .{ }^{1}$ Innovation in delivering effective clinical care to these people is therefore an urgent global priority. To reduce the risk of long term macrovascular and microvascular complications, ${ }^{2}$ UK, European, and US guidelines recommend early adoption of insulin as part of stepwise treatment intensification to bring glycated haemoglobin (HbA1c) below a general target of $7 \%$ (53 mmol/mol)..$^{3-6}$ Insulin initiation is often delayed, however, particularly in primary care, ${ }^{7}$ where 
implementation is not widespread despite being recommended as part of routine clinical management of type 2 diabetes. ${ }^{6}$ The mean HbA1c of people with type 2 diabetes before starting insulin is typically $1.5-2.0 \%$ above target: $9.3 \%$ (78 $\mathrm{mmol} / \mathrm{mol})$ in the UK, ${ }^{8} 8.6 \%$ (70 mmol/ mol) in a study in the US, ${ }^{9} 8.9 \%(74 \mathrm{mmol} / \mathrm{mol})$ in a large, multicountry primary care study, ${ }^{10}$ and 9.4\% (79 $\mathrm{mmol} / \mathrm{mol}$ ) in a community study in Australia (after a median diabetes duration of 8.1 years). ${ }^{11}$

Delay in treatment intensification by healthcare professionals, despite evidence that intensification is warranted and effective, ${ }^{12}$ is a major barrier to initiating insulin in people with type 2 diabetes. ${ }^{13}$ The delay can be due to health professional factors ${ }^{14}$ (for example, concerns about hypoglycaemia risk, lack of confidence or skills in insulin initiation or titration), health system factors $^{15}$ (competing priorities in busy, reactive primary care settings), and patient related factors (psychological resistance to insulin inititation ${ }^{16}$ ). Supporting and embedding insulin initiation in routine primary care practice is an important first step in potentially reducing referrals to costly secondary care, and in supporting timely, early optimisation of treatment, to better achieve glycaemic targets.

Our trial investigated the effectiveness of a new model of care: the Stepping Up model, which was designed to support insulin initiation in primary care among people with type 2 diabetes for whom it is clinically indicated. The Stepping Up model of care is built around an enhanced, reconfigured role for primary care practice nurses, ${ }^{17}$ who represent a rapidly growing section of the workforce and have a substantial role in the management of chronic conditions. Our approach was to train and mentor nurses to enhance their knowledge, skills, and confidence in discussing and implementing insulin initiation within the practice as a part of routine care. Furthermore, we aimed to set simple clinical protocols on insulin initiation. To implement these behaviour changes in professionals, we drew on normalisation process theory ${ }^{18}$ and the results of pilot studies. ${ }^{1719}$ Our aim was to address clinician and system level barriers to timely insulin initiation, and to normalise insulin initiation as part of standard primary care practice. We hypothesised that HbA1c would improve among participants in intervention arm practices, facilitated through timely insulin initiation, compared with the control arm. Furthermore, based on previous research, ${ }^{2021}$ we expected no major negative effect on participants' general emotional wellbeing (depressive symptoms).

\section{Methods}

Study design and participants

The study design and protocol have been described previously. ${ }^{22}$ In summary, we conducted a 12 month, two arm, non-blinded cluster randomised controlled trial, consistent with CONSORT guidelines, ${ }^{23}$ to investigate the effectiveness of the Stepping Up model of care compared with usual care.

General practices in Victoria, Australia, were eligible if they had at least one consenting general practitioner and practice nurse and could identify at least one eligible patient participant: adults with type 2 diabetes with above target $\mathrm{HbA1c}(\geq 7.5 \%$ (58 $\mathrm{mmol} / \mathrm{mol}))$ in the past six months who were already prescribed maximum oral treatment (at least two oral hypoglycaemia agents at maximum doses) or if their GP judged that insulin would be clinically appropriate. Patients were ineligible if they were aged more than 80 years, were already using insulin, had an estimated glomerular filtration rate $<30 \mathrm{~mL} / \mathrm{min} / 1.73 \mathrm{~m}^{2}$, were unable to give informed consent, or had a complex debilitating medical condition, such as severe mental illness, end stage cancer, or unstable cardiovascular disease.

Our original protocol was based on 58 practices and an average cluster size of 5. From our early recruitment experience and what was an achievable sample size, we subsequently revised this to 74 practices and an average cluster size of 3 (appendix files).

\section{Randomisation}

The unit of randomisation was the primary care practice. The study statistician computer generated stratified block randomisation sequences with varying block sizes $(4,6$, and 8 ) before recruitment. Practices were stratified by size ( $\leq 2$ versus $>2$ full time equivalent GPs), setting (private practice versus community health centre), and participation (or not) in type 2 diabetes quality improvement programmes (the Australian Primary Care Collaborative). After providing consent and recruiting at least one eligible patient, practices were randomised to intervention or usual care. We used this index case method $^{24}$ in all practices because our previous experience suggested that delaying randomisation of a cluster until all patients had been recruited risked loss of engagement of GPs. ${ }^{25}$ The research team then assisted practices to continue to identify and recruit patient participants (through searching the practice medical record database). This meant that allocation concealment after the index case was recruited was not possible for the GP and practice nurse. To minimise potential bias, participating patients were not informed of their study allocation until after they had provided consent. Blinding of GP, practice nurse, and patient was also not possible given the pragmatic nature of the intervention.

\section{Intervention}

The Stepping Up model of care, described elsewhere, ${ }^{1722}$ involved a reorientation of existing resources. Firstly, it included an enhanced role for the practice nurse in leading the discussion with patients about intensifying treatment through insulin initiation and titration. Secondly, the model of care set simple clinical protocols for insulin initiation and up-titration. Thirdly, the model of care reoriented the role of the specialist registered nurse with diabetes educator credentials in mentoring the practice nurse, rather than providing direct patient care. Intervention practices had an in-practice briefing and training session of 60-90 minutes for GPs and practice nurses, after which patients with confirmed eligibility and completed baseline data were invited to consult their GP for an assessment to discuss treatment 
intensification and referral to the practice nurse. Practice nurses did not prescribe insulin or manage insulin dosing without liaison with the GP, based on the legal scope of practice for generalist practice nurses in Australia.

Our model of care involved the acknowledgment and discussion with patients of the advantages and disadvantages of starting insulin treatment, including weight gain. We modelled shared decision making as a part of the intervention training, drawing on the principles of motivational interviewing. This set the scene for encouraging practitioners in intervention practices to approach participating patients with equipoise in relation to starting insulin. The intervention was necessarily brief in this pragmatic trial; however, we included in it guidance and checklists for GPs and practice nurses to discuss the pros and cons of insulin treatment and to elicit patient concerns and expectations, while also openly acknowledging and accepting that some patients may choose not to start insulin.

The role of the registered nurse with diabetes educator credentials in supporting and mentoring the practice nurse, and of the practice nurse in leading the discussion and implementation of insulin treatment with the patient in liaison with the GP, is outlined elsewhere. ${ }^{1520}$ Titration protocols were based on fasting blood glucose levels and use of a three day, 7 point blood glucose profile ${ }^{26}$ to identify the meal with the greatest postprandial excursion (see appendix files). We gave no additional instructions, so the GP had clinical autonomy regarding the management of oral hypoglycaemia agents. Practice nurses and GPs were encouraged to see patients as often as thought to be clinically appropriate over a period of up to 12 months, drawing as needed on the study registered nurse with diabetes educator credentials for mentoring and support, even if the patient remained undecided about, or had decided against, starting insulin. Further details about the intervention can be found in the referenced papers and appendices (see appendix files).

We gave control arm practices a copy of the Australian type 2 diabetes management guidelines ${ }^{27}$ and offered them training in the Stepping Up model of care after the 12 month follow-up of patient participants was complete.

\section{Patient involvement}

We sought feedback from participants after conducting an intervention pilot study ${ }^{15}$ and used this feedback to refine the model of care. We sought further participant feedback in a pilot of the data collection forms. Throughout the main trial we communicated with patient participants through a regular newsletter that included aggregate data about study progress and opportunities to provide feedback to the study team. We assessed the burden of the intervention on patients through interviews conducted at the end of the trial, as part of process evaluation (to be reported elsewhere). This evaluation was led by the Chronic Illness Alliance, a consumer advocacy organisation that has been a long term collaborator of our research group. We have thanked all participants for their involvement in the trial, and will provide, at a later date, a final summary report of the trial outcomes. Participants have access to the study website where all published results will be publicly available.

\section{Endpoints and data collection}

Our intervention targeted a process of care (insulin initiation); however, we chose a clinically meaningful disease outcome at an individual patient level as a primary endpoint: change (from baseline to 12 months) in HbA1c, measured as a continuous variable. We registered our primary outcome as an absolute HbA1c reduction of $0.5 \%$ in the intervention group compared with the control group. Measurement of HbA1c was performed at pathology laboratories aligned with the Diabetes Control and Complications Trial and the result was communicated to clinicians and patients as part of usual clinical care. Researchers retrieved these data from medical records or directly from pathology laboratories. Secondary endpoints included the proportion of participants who transitioned to insulin (this was amended from the original protocol where rate of insulin initiation was used, which proved impractical given the small cluster size in the study), the proportion who achieved a target $\mathrm{HbA} 1 \mathrm{c}$ of $\leq 7.0 \%(53 \mathrm{mmol} / \mathrm{mol})$ at 12 months, and change (from baseline to 12 months) in depressive symptoms (nine item patient health questionnaire, PHQ-9), ${ }^{28}$ diabetes specific distress (problem areas in diabetes scale, PAID), ${ }^{29}$ and generic health status (assessment of quality of life instrument, AQoL-8D). ${ }^{30}$ The Appendix files show differences between the registered outcomes reported here and those registered in the trial registry, and justification and explanation for any changes made. We also collected data on healthcare utilisation and costs, to be reported elsewhere.

All participants were provided with a blood glucose meter (Performa Nano; Roche Diagnostics) and instructed on its use. Subsidised low cost blood glucose testing strips were available through the National Diabetes Service Scheme. ${ }^{31}$ Data were uploaded from the meter at six and 12 months to a secure server.

\section{Statistical analysis}

Our statistical analysis plan has been published elsewhere. ${ }^{22}$ In brief, our sample size of 224 patients from 74 general practices (averaging three patients per practice) allowed us to detect an absolute $0.5 \%$ mean HbA1c difference at 12 months between control and intervention arms with $80 \%$ power and standard deviation of $1 \mathrm{using}$ a two sided $\alpha$ of 0.05. Data were analysed using Stata 13 (StataCorp, TX, USA). Descriptive statistics were used to summarise GP, practice nurse, and patient characteristics for the two study arms. Parametric data are reported as means (standard deviations) and non-parametric data as medians (interquartile ranges). Categorical data are reported as numbers (percentages). The individual patient was the unit of analysis and the analytical methods allow for clustering of patients within the practices. We compared binary outcomes between the 
two study arms with marginal logistic modelling using generalised estimating equations with robust standard errors and adjustment for baseline measures and clustering. Mixed effects linear regression was used to determine predictors for continuous outcomes, adjusting for baseline measures and clustering at the practice level. A $t$ test for proportions was used to compare the use of non-insulin agents between arms at 12 months, and a Wilcoxon ranked sum test was used to compare the number of days since insulin was started between control and intervention groups. Analyses were conducted on an intention to treat basis. All participants gave informed consent before enrolment.

\section{Results}

\section{Participating practices and patients}

Between October 2012 and January 2014, 93 primary care practices expressed interest and identified 521 potentially eligible patients (fig 1). Subsequently, 19 practices did not consent any eligible patients, leaving 74 participating practices for randomisation. Two hundred and 50 five of the potentially eligible patients were subsequently found to be ineligible at screening $(n=156)$ or did not respond to the invitation letter $(n=99)$. By April 2014, the 74 practices had identified and consented 266 eligible participants ( $73 \%$ of potentially eligible patients identified).

Table 1 shows the baseline characteristics of practices, GPs, practice nurses, and participants with type 2 diabetes. Of the total sample, 248 (93\%) completed the 12 month follow-up for the primary endpoint. No differences in baseline characteristics were observed between study completers and non-completers, except for a higher proportion of women not completing than men $(11 v 7)$.

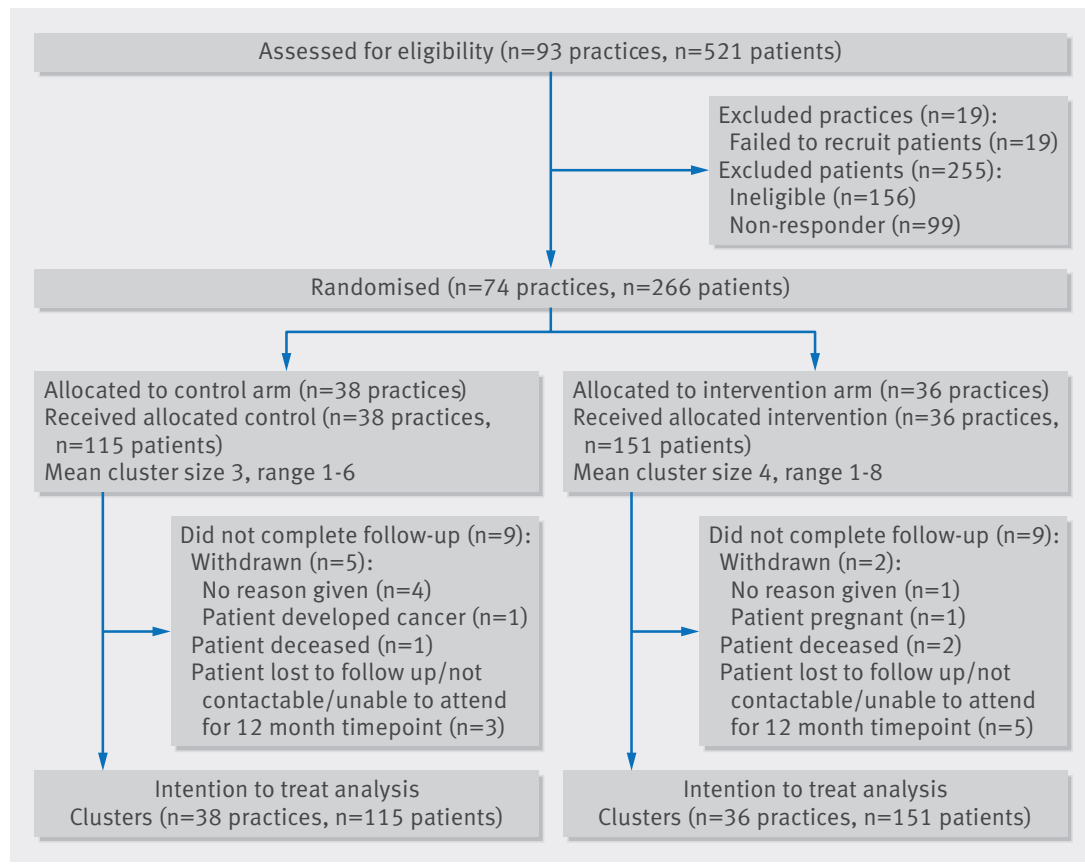

Fig 1 | CONSORT diagram for the Stepping Up randomised control trial

\section{Primary and secondary endpoints}

Table 2 shows the primary and secondary endpoints. At 12 months there was a statistically and clinically significant difference between study arms in terms of change in HbA1c (mean difference: $-0.6 \%$, 95\% confidence interval $-0.9 \%$ to $-0.3 \%, \mathrm{P}<0.001$ ), favouring the intervention. This is consistent with achieving our registered primary outcome of an absolute HbA1c reduction of $0.5 \%$ in the intervention group compared with the control group. The majority of this change in HbA1c seen in both arms was achieved by six months (fig 2).

In the intervention arm, 105/151 (70\%) patients started insulin (102 were using insulin at 12 months), whereas in the control arm, 25/115 (22\%) started insulin (24 were using insulin at 12 months). The median number of days from baseline assessment to insulin initiation in intervention and control group patients who started insulin was 32 (interquartile range 11.5-134.5) days and 85 (63-191) days, respectively (statistically significant difference: two sample Wilcoxon rank sum test; $\mathrm{P}=0.005)$. In the intervention arm, 17 (11\%) patients had started rapid acting insulin at 12 months, compared with one patient in the control arm $(\mathrm{P}<0.001)$. Further data on insulin use in participants are available in the appendix files.

Target HbA1c ( $\leq 7 \%$ (53 mmol $/ \mathrm{mol})$ ) was achieved by 54 (36\%) intervention participants (32 of whom were using insulin at 12 months) and 22 (19\%) control participants (two of whom had started insulin): odds ratio 2.2 (95\% confidence interval 1.2 to 4.3 ), $\mathrm{P}=0.02$. Twenty two (15\%) intervention participants and 20 (17\%) control participants achieved target HbA1c without starting insulin.

At 12 months, depressive symptoms had not worsened and there was no statistically significant difference between arms in the mean change (patient health questionnaire-9: -1.1 (SD 3.5) $v-0.1$ (2.9)). There was a statistically significant difference in mental health (AQoL mental component summary: 0.04 (0.16) $v-0.002$ (0.13)), favouring the intervention, but no significant difference in physical health (AQoL physical component summary: $0.03(0.15) v 0.02(0.13))$ or diabetes specific distress (PAID: -5.6 (15.5) v-2.4 (15.4)). There was no statistically significant difference between arms in the proportion of participants experiencing moderate to severe depressive symptoms or severe diabetes specific distress (table 2), nor was there any difference by insulin initiation (Wilcoxon rank sum test; $\mathrm{P}=0.98$ ). No significant difference was found in PHQ-9, AQoL, or PAID scores when comparing participants who started insulin with those who did not (data not shown).

At 12 months there was an average weight gain in the intervention arm and an average weight loss in the control group (1.7 (SD 5.2) kg $v-1.1$ (5.1), mean difference 2.8 ( $95 \%$ confidence interval 1.5 to 4.0 ) $\mathrm{kg}$ ). There were no statistically significant differences in blood pressure or other biochemical measures between arms at follow-up, with the exception of triglycerides, which remained higher in the control group.

At baseline, participants were using a mean of 2.0 (SD 0.6) classes of non-insulin hypoglycaemic agents, with 


\begin{tabular}{|c|c|c|}
\hline Characteristics & Intervention arm & Control arm \\
\hline \multicolumn{3}{|l|}{ Practices } \\
\hline Primary care practices & $36(49)$ & $38(51)$ \\
\hline \multicolumn{3}{|l|}{ Type of practice: } \\
\hline Private practice & $27(75.0)$ & $31(81.6)$ \\
\hline Corporate practice & $7(19.4)$ & $5(13.2)$ \\
\hline Community health centre & $2(5.6)$ & $2(5.3)$ \\
\hline \multicolumn{3}{|l|}{ Location of practice: } \\
\hline Major city & $26(72.2)$ & $21(55.3)$ \\
\hline Inner regional area & $9(25.0)$ & $13(34.2)$ \\
\hline Outer regional area & $1(2.8)$ & $4(10.5)$ \\
\hline Median (IQR) No of physicians per practice & $5(4-9.5)$ & $5(4-9)$ \\
\hline Median (IQR) No of practice nurses per practice & $2.5(2-3.5)$ & $2(1-4)$ \\
\hline Registered nurse with credentials for diabetes educator on site & $12(33.3)$ & $14(36.8)$ \\
\hline Median (IQR) No of patients per full time equivalent general practitioner ${ }^{\star} \dagger$ & $1738(1176-2727)$ & $1316(911-1726$ \\
\hline General practitioners & $83(51.2)$ & $79(48.8)$ \\
\hline Mean (SD) age (years) $\ddagger$ & $48.8(9.9)$ & $49.7(11.2)$ \\
\hline Women & $34(41.0)$ & $27(34.2)$ \\
\hline Working hours/week $\ddagger$ & $36.6(10.5)$ & $37.3(11.6)$ \\
\hline Median (IQR) years of experience & $19(8-26)$ & $20(7-30)$ \\
\hline Experience with insulin initiation in preceding 12 months§ & $48(60.0)$ & $36(46.2)$ \\
\hline Practice nurses & $48(46.6)$ & $55(53.4)$ \\
\hline Mean (SD) age (years)ף & $44.7(10.2)$ & $46.0(9.9)$ \\
\hline Women & $48(100)$ & $55(100)$ \\
\hline Diabetes educator training & $6(12.5)$ & $7(12.7)$ \\
\hline Experience with insulin initiation in preceding 12 months & $16(33.3)$ & $16(29.1)$ \\
\hline Adults with type 2 diabetes & $151(56.8)$ & $115(43.2)$ \\
\hline Mean (SD) age (years) & $61.7(9.7)$ & $62.0(10.6)$ \\
\hline Women & $62(41.1)$ & $41(35.7)$ \\
\hline \multicolumn{3}{|l|}{ Highest level of education: } \\
\hline Primary or less & $14(9.3)$ & $12(10.4)$ \\
\hline Secondary or trade & $101(66.9)$ & $83(72.2)$ \\
\hline Tertiary & $36(23.8)$ & $20(17.4)$ \\
\hline Employed & $67(44.4)$ & $50(43.5)$ \\
\hline Healthcare card holder & $75(49.7)$ & $62(53.9)$ \\
\hline Median (IQR) diabetes duration (years) & $8(5-12)$ & $9(5-14)$ \\
\hline Median (IQR) HbA1c (\%) & $8.7(8.1-9.7)$ & $8.5(8-9.6)$ \\
\hline Median (IQR) HbA1c (mmol/mol) & $72(65-83)$ & $69(64-81)$ \\
\hline Median (IQR) No of medical conditions & $3(2-5)$ & $3(2-5)$ \\
\hline Median (IQR) No of drugs & $6(5-10)$ & $7(5-10)$ \\
\hline Median (IQR) drug adherence rating scale ${ }^{\star \star}$ & $29(26-30)$ & $29(27-30)$ \\
\hline \multicolumn{3}{|l|}{ Diabetes complicationstt: } \\
\hline Microvascular & $17(11.3)$ & $16(13.9)$ \\
\hline Macrovascular & $22(14.6)$ & $21(18.3)$ \\
\hline Total cholesterol(mmol/L) $\ddagger \ddagger$ & $4.3(1.0)$ & $4.2(1.1)$ \\
\hline Triglycerides $(\mathrm{mmol} / \mathrm{L}) \dagger \ddagger \ddagger$ & $1.9(0.1)$ & $2.3(1.4)$ \\
\hline LDL cholesterol (mmol/L)§§ & $2.3(0.9)$ & $2.1(0.9)$ \\
\hline HDL cholesterol $(\mathrm{mmol} / \mathrm{L})$ ๆๆ & $1.2(0.3)$ & $1.1(0.3)$ \\
\hline Estimated glomerular filtration rate ${ }^{\star \star \star}$ & $79.4(14.4)$ & $78.8(14.6)$ \\
\hline \multicolumn{3}{|l|}{ Blood pressure $(\mathrm{mm} \mathrm{Hg})$ : } \\
\hline Systolic & $134.6(15.7)$ & $133.5(15.2)$ \\
\hline Diastolic & $79.6(11.1)$ & $78.5(9.5)$ \\
\hline \multicolumn{3}{|l|}{ 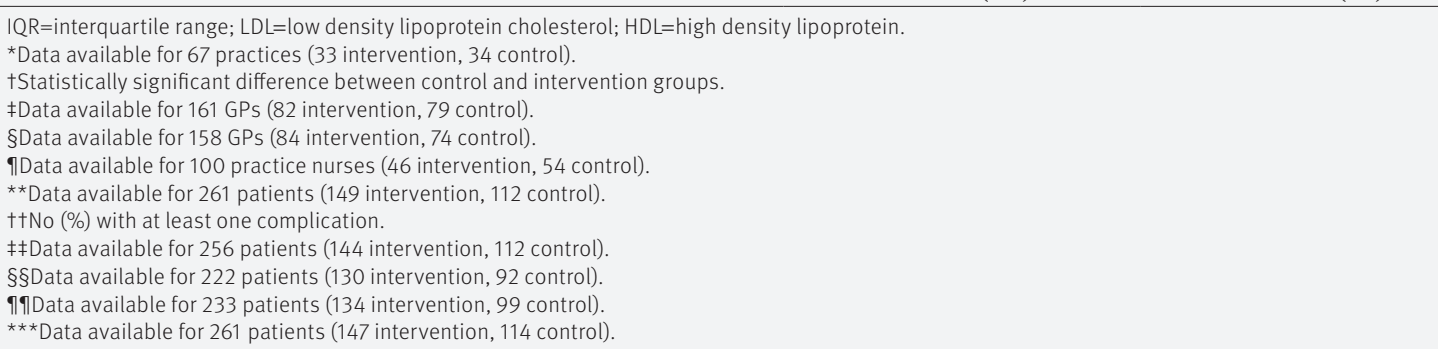 } \\
\hline
\end{tabular}




\begin{tabular}{|c|c|c|c|c|}
\hline \multirow[b]{2}{*}{ Endpoints } & \multirow[b]{2}{*}{ Intervention arm } & \multirow[b]{2}{*}{ Control arm } & \multicolumn{2}{|c|}{$\begin{array}{l}\text { Adjusted data for baseline } \\
\text { measure and clustering }\end{array}$} \\
\hline & & & $\begin{array}{l}\text { Treatment effect } \\
(95 \% \mathrm{Cl})\end{array}$ & Pvalue \\
\hline \multicolumn{5}{|l|}{ HbA1c (\%): } \\
\hline Baseline & $8.7(8.1-9.7)$ & $8.5(8-9.6)$ & & \\
\hline Follow-up & $7.4(6.9-8.2)$ & $8.0(7.1-9.0)$ & & \\
\hline Change & $-1.3(1.4)$ & $-0.6(1.5)$ & $-0.6(-0.9$ to -0.3$)$ & $<0.001$ \\
\hline \multicolumn{5}{|c|}{ No (\%) of participants using insulin: } \\
\hline Follow-up & $105(69.5)$ & $25(21.7)$ & $8.3^{\star}(4.5$ to 15.4$)$ & $<0.001$ \\
\hline \multicolumn{5}{|c|}{ No (\%) of participants with $\mathrm{HbA} 1 \mathrm{c} \leq 53 \mathrm{mmol} / \mathrm{mol}(7 \%)$ : } \\
\hline Follow-up & $54(35.8)$ & $24(20.9)$ & $2.2^{\star}(1.2$ to 4.3$)$ & 0.02 \\
\hline \multicolumn{5}{|c|}{ Depressive symptoms (PHQ-9)t: } \\
\hline Baseline & $3(1-7)$ & $2(1-6.5)$ & & \\
\hline Follow-up & $2(0-5)$ & $2(0-5)$ & & \\
\hline Change & $-1.1(3.5)$ & $-0.1(2.9)$ & $-0.8(-1.6$ to -0.01$)$ & 0.047 \\
\hline \multicolumn{5}{|c|}{ No (\%) of participants with moderate-severe depressive symptoms (PHQ-9 total: $\geq 10) \neq:$} \\
\hline Baseline & $22(15.1)$ & $15(13.5)$ & & \\
\hline Follow-up & $19(12.8)$ & $15(13.3)$ & $0.82^{\star}(0.3$ to 2.2$)$ & 0.69 \\
\hline \multicolumn{5}{|c|}{ Diabetes specific distress (PAID)§: } \\
\hline Baseline & $15(6.3-31.3)$ & $12.5(5-23.8)$ & & 0.14 \\
\hline Follow-up & $8.8(3.8-22.5)$ & $10(2.5-23.8)$ & & \\
\hline Change & $-5.6(15.5)$ & $-2.4(15.4)$ & $-1.9(-5.1$ to 1.3$)$ & 0.24 \\
\hline \multicolumn{5}{|c|}{ No (\%) of participants with severe diabetes specific distress (PAID total: $\geq 40) \neq$ : } \\
\hline Baseline & $25(16.8)$ & $14(12.4)$ & & \\
\hline Follow-up & $18(12.1)$ & $12(10.4)$ & $1.0 *(0.4$ to 2.3$)$ & 0.93 \\
\hline \multicolumn{5}{|c|}{ Health status (AQoL-8D) physical component scoref: } \\
\hline Baseline & $0.63(0.20)$ & $0.61(0.21)$ & & \\
\hline Follow-up & $0.66(0.21)$ & $0.64(0.21)$ & & 0.52 \\
\hline Change & $0.03(0.15)$ & $0.02(0.13)$ & $0.01(-0.03$ to 0.04$)$ & 0.64 \\
\hline \multicolumn{5}{|c|}{ Health status (AQoL-8D) mental component scoref: } \\
\hline Baseline & $0.45(0.20)$ & $0.45(0.22)$ & & \\
\hline Follow-up & $0.48(0.21)$ & $0.45(0.22)$ & & \\
\hline Change & $0.04(0.16)$ & $-0.002(0.13)$ & $0.04(0.002$ to 0.08$)$ & 0.04 \\
\hline \multicolumn{5}{|l|}{ Weight (kg): } \\
\hline Baseline & $90.8(19.6)$ & 94.6 (18.9) & & \\
\hline Follow-up & $92.5(20.1)$ & 93.5 (18.9) & & \\
\hline Change & $1.7(5.2)$ & $-1.1(5.1)$ & 2.8 (1.5 to 4.0$)$ & $<0.001$ \\
\hline
\end{tabular}

*Odds ratio.

+Patient health questionnaire 9. Range of possible scores: 0 -27. A total score of $\geq 10$ indicates at least moderate depressive symptoms. Data available for 261 patients at baseline (149

intervention, 112 control) and 263 at 12 months (149 intervention, 114 control; intention to treat (ITT) population).

‡Data available for 257 patients at baseline (146 intervention, 111 control) and 261 at 12 months (148 intervention, 113 control; ITT).

§Problem areas in diabetes. Range of possible scores: 0-100. A score of $\geq 40$ indicates severe diabetes related distress. Data available for 262 patients at baseline (149 intervention, 113 control) and 264 at 12 months (149 intervention, 115 control; ITT).

१Assessment of quality of life. Maximum possible score is 1 . Higher scores indicate better generic health status. Data available for 262 patients at baseline (149 intervention, 113 control) and 263 at 12 months (149 intervention, 114 control; ITT).

no significant difference between arms ( $t$ test, $\mathrm{P}=0.89$ ). The majority of patients were prescribed metformin (93\% across both arms) and sulfonylureas (63\% across both arms). There was no significant difference in the prescription of individual drug classes by study arm. The mean number of classes of non-insulin hypoglycaemic agents being used at 12 months was higher in the control arm compared with the intervention arm (2.3 (SD $0.1) v 1.9(0.1) ; \mathrm{P}=0.01)$. A higher proportion of people in the control group used dipeptidyl peptidase 4 inhibitors than in the intervention group at 12 months (table 3 ).

Practices in the intervention arm received 183 mentoring support visits from the study registered nurse with diabetes educator credentials (mean visits per practice 5.2 (range 1-8)). Thirty two per cent (48/151) of participants in intervention practices completed at least one three day, 7 point structured blood glucose monitoring profile over the 12 month study. Practice nurses estimated the time they spent on the study (clinical interactions with participating patients and research tasks). On a per practice basis, 23 control practices and 27 intervention practices reported a median of 1.5 (interquartile range 0-3.6) hours and 18 (9-20.9) hours, respectively.

Overall, 58\% of people in the control group were recruited before practice randomisation compared with $45 \%$ in the intervention group (significant difference $\mathrm{P}=0.033)$. Sensitivity analysis was conducted to explore whether there was any difference at baseline between patients who were recruited before and after randomisation of the practices. No statistically significant differences were found. Mixed effects linear regression 


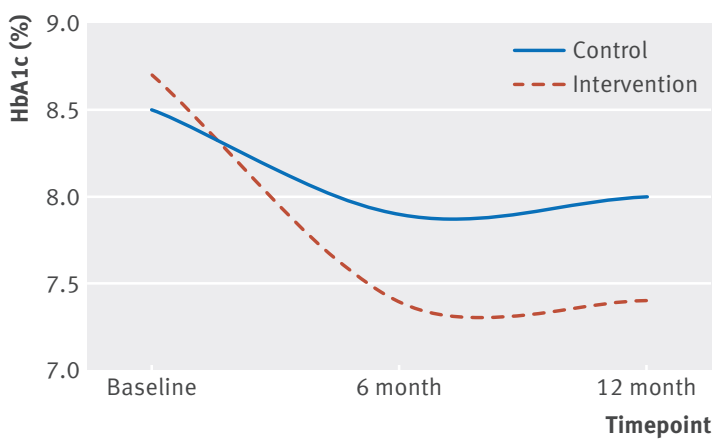

Fig $2 \mid$ Change in primary endpoint at six and 12 months. $\mathrm{HbA1c}=$ glycated haemoglobin

was used to determine the impact of the intervention, adjusting for clustering at the practice level for each of these groups. Treatment effect in terms of the primary outcome remained significant in both groups (treatment effect: -0.57 (95\% confidence interval -1.1 to -0.05 ), $\mathrm{P}=0.03$ and -0.98 ( -1.49 to -0.48$), \mathrm{P}<0.001$ for patients assessed before and after randomisation, respectively).

\section{Adverse events}

No severe hypoglycaemic events (those that would require third party assistance for recovery) or other adverse events were reported in either study arm.

\section{Discussion}

Our model of care changed clinical practice, with most participants in the intervention arm starting insulin, producing a clinically and statistically significant improvement in glycaemia among adults with type 2 diabetes managed in primary care. This was despite a higher patient to GP ratio in intervention practices, and was achieved safely, with no severe hypoglycaemic events, and without deterioration in emotional wellbeing or health status. Our results indicate that, with appropriate support and redesign of the practice system, insulin initiation can become part of routine diabetes management in primary care, obviating the need to refer to specialist services with geographical, cost, and accessibility barriers.

\section{Strengths and limitations of this study}

A strength of our study is the robust theoretical and empirical base to our intervention. Our pragmatic trial

\begin{tabular}{|c|c|c|c|}
\hline Drug classes & $\begin{array}{l}\text { No }(\%) \text { in } \\
\text { intervention } \\
\operatorname{arm}(n=146)\end{array}$ & $\begin{array}{l}\text { No }(\%) \text { in } \\
\text { control arm } \\
(n=108)\end{array}$ & Pvalue* \\
\hline Metformin & $133(91.1)$ & $96(88.9)$ & 0.56 \\
\hline Sulphonylurea & $75(51.4)$ & $64(59.3)$ & 0.21 \\
\hline Acarbose & $3(2.1)$ & $2(1.9)$ & 0.91 \\
\hline Dipeptidyl peptidase 4 inhibitorst & $25(17.1)$ & $38(35.2)$ & 0.001 \\
\hline Glitazones & $6(4.1)$ & $5(4.6)$ & 0.8411 \\
\hline Sodium-glucose cotransporter 2 inhibitors & $2(1.4)$ & $2(1.9)$ & 0.76 \\
\hline Glucagon-like peptide 1 agonists & $9(6.2)$ & $7(6.5)$ & 0.92 \\
\hline
\end{tabular}

*t test of proportions.

tAt time of the trial dipeptidyl peptidase 4 inhibitors were not subsidised for use with insulin. of a complex intervention addressed several known barriers to overcoming delay in starting insulin treatment. For example, an intentional component of our system redesign was reorienting the practice nurse and registered nurse with diabetes educator credentials roles, allowing additional time to be spent with patients, within existing resources. Other strengths include the cluster randomised design, minimising the risk of contamination, and our excellent rate of participant retention (93\%).

Our study had limitations. Firstly, practices were randomised after the first consenting patient was identified, raising the possibility of selection bias. However, the balance in key patient characteristics between the study arms means that any such bias was minimal. Secondly, while a smaller cluster size is generally preferable in a cluster randomised trial, the relatively large variation in the cluster sizes in our study may make statistical adjustments for clustering less effective, in particular when the number of clusters is small. Thirdly, our sample may not have been fully representative of the broader population of adults with type 2 diabetes managed in primary care for whom insulin is clinically indicated. Overall, less than $15 \%$ of our sample had severe diabetes specific distress or moderate to severe depressive symptoms, a lower rate than in a recent national Australian sample. ${ }^{32}$ We will explore implementation fidelity and variation in more detail through a qualitative process evaluation in a subsequent paper. Finally, our drugs and hypoglycaemia data were derived from GP records and subject to the same accuracy limitations of any routinely collected clinical dataset. Hypoglycaemia is typically underestimated ${ }^{33}$ and is likely to be under-reported in routine medical records. ${ }^{34}$ In particular, severe hypoglycaemia is serious but relatively rare and may not have been detected in our study, given our sample size.

\section{Comparison with other studies}

Only two other trials have tested interventions to change clinical practice in this way. The AIM@GP trial showed no improvement in insulin prescribing rates or glycated haemoglobin. ${ }^{35}$ It provided scheduled and ongoing telephone support from a specialist diabetes educator and the option to refer patients to an offsite community pharmacist for a one hour insulin initiation session. Our intervention differed in that it was based completely in the familiar environs of patients' own primary care practices, built on existing relationships and resources (with the practice based practice nurses), and provided an immediate pathway for the GP to delegate this clinical task. A UK study, using a before and after evaluation design, showed improved HbA1c $(-1.4 \%)$ at six months in patients who started insulin, ${ }^{36}$ similar in magnitude to the improvement in our study. That intervention combined education with face to face and telephone support from a registered nurse with diabetes educator credentials for GPs and practice nurses and involved full day, offsite training for both GPs and practice nurses. In contrast, our intervention used a brief (60-90 minute) onsite training session incorporated 
into the daily running of the practice with flexible support from the registered nurse with diabetes educator credentials, as required. Consistent with previous research, ${ }^{37}$ our study participants did not report worse psychological outcomes at follow-up, suggesting that insulin treatment can be initiated in primary care for people with type 2 diabetes without impairing their emotional wellbeing. In fact, at 12 months, the reduction in depressive symptoms in intervention arm participants was significantly greater than that in the control arm. Insulin initiation (across both arms) did not affect depressive symptoms, diabetes specific distress, or generic health status at 12 months.

\section{Conclusions and policy and practice implications}

Our pragmatic trial findings have important implications for the organisation of healthcare and for health policy. Our model of care is based on an enhanced role for a practice nurse and would not be feasible where primary care doctors work in solo practice without access to a practice nurse. Nevertheless, even in high resource settings, where the move to multidisciplinary primary healthcare teams is growing-for example, through the growth of the Medical Home movement, ${ }^{38}$ our study suggests that simply having access to a practice nurse will not increase the rate of appropriate insulin initiation. To make the best use of resources, primary care workforce models need to be developed and implemented to reorient the way specialists (registered nurse with diabetes educator credentials and endocrinologists) offer support to primary care teams that include well supported and resourced primary care nurses. Changes in the role of registered nurse with diabetes educator credentials is occurring in some health systems,38 and our study provides evidence of the effectiveness and safety of such models of care. Rather than waiting for referrals, specialist services need to offer proactive, tailored secondary consultation, liaison, and mentoring services that are flexible and supportive of the needs of primary care practitioners and patients. Scaling up the model of care in metropolitan centres would require engaging with hospitals and other health services in reorienting the role of registered nurse with diabetes educator credentials currently employed in direct patient care. Investigation of e-health modalities (for example, online training, support, and video consultations) could support implementation of this model of care in more distant rural or remote settings.

Our trial findings also have implications for clinical practice. The issue around personalising glycaemic targets and treatments is an important and emerging consideration in the care of people with type 2 diabetes. When our trial started, there was vigorous debate about the need for caution in setting lower targets. In addition, our exclusion criteria ruled out participants for whom a higher target would definitely be considered. Collecting reliable data on duration of cardiovascular disease, severe hypoglycaemia, and hypoglycaemic unawareness did not prove feasible in this setting. We therefore decided to use the general target of $7 \%$. Debate continues about the advantages and disadvantages of intensifying treatment for people with type 2 diabetes who have the HbA1c levels mandated in our study. Our findings suggest that the Stepping Up model of care builds clinical capacity within GP and practice nurse teams to undertake the work of insulin initiation. However, the model of care did not mandate a dogmatic approach to such changes in treatment. It is worth noting the major change in HbA1c was achieved at six months, and that even in intervention practices, only $35 \%$ of participants with type 2 diabetes achieved the general $\mathrm{HbA1c}$ target of $<7 \%$ (53 $\mathrm{mmol} / \mathrm{mol})$, suggesting that practitioners and patients were judicious in the way they approached progressive treatment intensification, within the new model of care.

Our clinical protocols and algorithms were focused solely on insulin. It is worth noting that NPH (neutral protamine hagedorn) insulin remains widely used and that the added costs of analogueue insulins where NPH can be used without problem is still subject to debate. While we did not prespecify weight gain as a secondary outcome, this is an adverse effect of insulin treatment and we have chosen to report it. While insulin is still regarded as an essential treatment option, as the range of glycaemic treatments grows, clinical algorithms become more complex. ${ }^{5}$ Future research needs to explore the capacity to generate recommendations for real time personalised treatment intensification that incorporate this increasing complexity, ${ }^{39}$ to be used as part of the practice nurse led model of care. Future research could also address the extent to which improvements in glycaemic levels are maintained, and the extent to which the model of care is sustained in routine clinical practice. In particular, research could explore use of the model of care to specifically support early adoption of insulin treatment to achieve glycaemic targets early in people with recently diagnosed type 2 diabetes.

The global epidemic of type 2 diabetes demands innovation in care delivery. Delaying insulin initiation when clinically indicated is neither ethical nor effective. Furthermore, health systems will not cope with demand if insulin initiation remains anchored in specialist centres, nor will they be able to respond to the imperative to achieve glycaemic targets early in people with recently diagnosed type 2 diabetes. Thus our pragmatic, translational study has important implications across health systems globally for the organisation of care for people with type 2 diabetes. Our effective model of care has the potential to improve outcomes while making better use of scarce healthcare resources.

\section{AUTHOR AFFILIATIONS}

${ }^{1}$ Department of General Practice, University of Melbourne, Carlton, Melbourne, VIC, 3053, Australia

2Department of Medicine, St Vincent's Hospital, University of Melbourne, Melbourne, Australia

${ }^{3}$ School of Psychology, Deakin University, Victoria, Australia

4The Australian Centre for Behavioural Research in Diabetes, Diabetes Victoria, Victoria, Australia

${ }^{5}$ AHP Research, United Kingdom

${ }^{6}$ Melbourne EpiCentre, the University of Melbourne, Melbourne, Australia 
${ }^{7}$ Faculty of Medicine, Dentistry and Health Sciences, University of Melbourne, Australia

${ }^{8}$ Lee Kong Chian School of Medicine, Nanyang Technological University, Singapore

${ }^{9}$ School of Nursing, University of Melbourne, Australia

${ }^{10} \mathrm{~S} c h$ ool of Public Health and Preventive Medicine, Monash University, Australia

${ }^{11}$ Health Economics and Social Policy Group, Division of Health

Sciences, University of South Australia, Adelaide, Australia

${ }^{12}$ Faculty of Health Sciences, University of Southampton,

Southampton, UK

13John Richards Initiative, Australian Institute for Primary Care and Ageing, College of Science, Health and Engineering, La Trobe University, Melbourne, Australia

We thank the general practitioners, practice nurses, and people with type 2 diabetes who participated in the study.

Contributors: JF, IB, DY, and JB conceptualised the original study proposal and secured funding. JF had overall responsibility for the study. JF, DON, JS, JMN, EHT, LG, DY, JB, EP, DL, LS, CM, and IB drafted the protocol. LG also contributed to data collection. JF, DON, JS, JMN, $A G, E H T, D L$, and IB contributed to the statistical analysis plan and JMN and AG led the data analysis. JF wrote the original draft of the report. All authors contributed to data interpretation and approved the final report. As corresponding author and principal investigator, JF had full access to all the data in the study and takes responsibility for the integrity of the data and the accuracy of the data analysis. JF had final responsibility for the decision to submit for publication. He is the guarantor.

Funding: The study was funded by the Australian National Health and Medical Research Council (ID 1023738) and was supported by an educational/research grant by Roche Diagnostics Australia.

Competing interests: All authors have completed the ICMJE uniform disclosure form at www.icmje.org/coi_disclosure.pdf and declare: We acknowledge funding from the Australian National Health and Medical Research Council (project grant application: APP1023738). The study was also supported by an educational/research grant by Roche Diabetes Care, the RACGP Foundation RACGP/Independent Practitioner Network Grant and received in-kind support from Sanofi. Xclinical hosted the BG data. IF was supported by a National Health and Medical Research Council Career Development Fellowship. IS is supported by core funding to the Australian Centre for Behavioural Research in Diabetes from Diabetes Victoria and Deakin University. JMN was supported by a National Health and Medical Research Council postgraduate scholarship. EHT is supported by an Australian postgraduate award Deakin University PhD scholarship. IF has received unrestricted educational grants for research support from Roche Diabetes Care, Sanofi, and Medtronic; JS is a member of the Accu-Check Advisory Board (Roche Diabetes Care). Her research group (ACBRD) has received unrestricted educational grants from Medtronic and Sanofi Diabetes; sponsorship to host or attend educational meetings from Lilly, Medtronic, MSD, Novo Nordisk, Roche Diabetes Care, and Sanofi Diabetes; consultancy income from Abbott Diabetes Care, Astra Zeneca, Roche Diabetes Care, and Sanofi Diabetes; DNO, DL and JMN had various financial relationships with pharmaceutical industries outside the submitted work including consultancies, grants, lectures, educational activities, and travel. DNO has received research and travel support and honorariums from Sanofi, Roche and Novo and is an advisory board member to Sanofi, Novo, and Abbott. JMN has no financial relationships with companies marketing blood glucose monitoring devices, but has received payment from Sanofi who funded the control practice training at end of study. DL has received honorariums and research grants from Sanofi Australia. The study sponsors had no role in the design and conduct of the study; collection, management, analysis, and interpretation of the data; and preparation, review, or approval of the manuscript.

Ethical approval: The study protocol was approved by the University of Melbourne Health Sciences human research ethics subcommittee (ID 123740) and registered with the Australian and New Zealand Clinical Trials Registry (ACTRN12612001028897).

Data sharing: Anonymised patient level data are available on reasonable request from the authors.

Transparency: The manuscript's guarantor (JF) affirms that the manuscript is an honest, accurate, and transparent account of the study being reported; that no important aspects of the study have been omitted; and that any discrepancies from the study as planned have been explained.
This is an Open Access article distributed in accordance with the Creative Commons Attribution Non Commercial (CC BY-NC 4.0) license, which permits others to distribute, remix, adapt, build upon this work non-commercially, and license their derivative works on different terms, provided the original work is properly cited and the use is non-commercial. See: http://creativecommons.org/licenses/ by-nc/4.0/.

1 Zimmet PZ, Magliano DJ, Herman WH, Shaw JE. Diabetes: a 21st century challenge. Lancet Diabetes Endocrinol 2014;2:56-64. doi:10.1016/S2213-8587(13)70112-8

2 Holman RR, Paul SK, Bethel MA, Matthews DR, Neil HA. 10-year follow-up of intensive glucose control in type 2 diabetes. N Engl / Med 2008:359:1577-89 doi:10.1056/NEIMoa0806470.

3 Inzucchi SE, Bergenstal RM, Buse JB, et al. American Diabetes Association (ADA) European Association for the Study of Diabetes (EASD). Management of hyperglycemia in type 2 diabetes: a patient-centered approach: position statement of the American Diabetes Association (ADA) and the European Association for the Study of Diabetes (EASD). Diabetes Care 2012;35:1364-79. doi:10.2337/dc12-0413.

4 Nathan DM, Buse JB, Davidson MB, et al. Management of hyperglycemia in type 2 diabetes: a consensus algorithm for the initiation and adjustment of therapy: update regarding thiazolidinediones: a consensus statement from the American Diabetes Association and the European Association for the Study of Diabetes. Diabetes Care 2008:31:173-5. doi:10.2337/dc08-9016.

5 American Diabetes Association. Standards of Medical Care in Diabetes-2016 Abridged for Primary Care Providers. Clin Diabetes 2016;34:3-21. doi:10.2337/diaclin.34.1.3.

6 McGuire H, Longson D, Adler A, Farmer A, Lewin I. Guideline Development Group. Management of type 2 diabetes in adults summary of updated NICE guidance. BMJ 2016;353:11575. doi:10.1136/bmj.i1575.

7 Shah BR, Hux JE, Laupacis A, Zinman B, van Walraven C. Clinical inertia in response to inadequate glycemic control: do specialists differ from primary care physicians?Diabetes Care 2005;28:600-6. doi:10.2337/ diacare.28.3.600.

8 Blak BT, Smith HT, Hards M, Maguire A, Gimeno V. A retrospective database study of insulin initiation in patients with Type 2 diabetes in UK primary care. Diabet Med 2012;29:e191-8. doi:101111/j.1464-5491 2012.03694.x

9 Curtis B, Lage MJ. Glycemic control among patients with type 2 diabetes who initiate basal insulin: a retrospective cohort study. I Med Econ 2014:17:21-31. doi:10.3111/13696998.2013.862538.

10 Khunti K, Damci T, Meneghini L, Pan CY, Yale JF. SOLVE Study Group. Study of Once Daily Levemir (SOLVETM): insights into the timing of insulin initiation in people with poorly controlled type 2 diabetes in routine clinical practice. Diabetes Obes Metab 2012;14:654-61. doi:10.1111/j.1463-1326.2012.01602.x

11 Davis TME, Davis Cyllene Uwa Edu Au WA, Bruce DG. Glycaemic levels triggering intensification of therapy in type 2 diabetes in the community: the Fremantle Diabetes Study. Med J Aust 2006;184:325-8

12 Phillips LS, Branch WT, Cook CB, et al. Clinical inertia. Ann Intern Med 2001;135:825-34 doi:10.7326/0003-4819-135-9-200111060-00012.

3 Khunti S. Davies M, Khunti K. Clinical inertia in the management of type 2 diabetes mellitus: a focused literature review. Br J Diabetes Vasc Dis 2015;15:65-9doi:10.15277/bjdvd.2015.019.

14 Kunt T, Snoek FJ. Barriers to insulin initiation and intensification and how to overcome them. Int / Clin Pract Suppl 2009;63:6-10. doi:10.1111/j.1742-1241.2009.02176.x.

15 Furler J, Spitzer O, Young D, et al. Barriers and enablers to timely initiation of insulin in General Practice. Aust Fam Physician 2011:40:617-23

16 Polonsky WH, Fisher L, Guzman S, Villa-Caballero L, Edelman SV. Psychological insulin resistance in patients with type 2 diabetes: the scope of the problem. Diabetes Care 2005;28:2543-5. doi:10.2337| diacare.28.10.2543.

17 Furler JS, Blackberry ID, Walker C, et al. Stepping up: a nurse-led model of care for insulin initiation for people with type 2 diabetes. Fam Pract 2014:31:349-56. doi:10.1093/fampra/cmt085.

18 May CR, Mair F, Finch T, et al. Development of a theory of implementation and integration: Normalization Process Theory. Implement Sci 2009:4:29. doi:10.1186/1748-5908-4-29.

19 Blackberry ID, Furler IS, Ginnivan LE, et al. An exploratory trial of basa and prandial insulin initiation and titration for type 2 diabetes in primary care with adjunct retrospective continuous glucose monitoring: INITIATION study. Diabetes Res Clin Pract 2014;106:24755. doi:10.1016/j.diabres.2014.08.011.

20 Hajos TRS, Pouwer F, de Grooth R, et al. Initiation of insulin glargine in patients with Type 2 diabetes in suboptimal glycaemic control positively impacts health-related quality of life. A prospective cohort study in primary care. Diabet Med 2011;28:1096-102. doi:10.1111/j.1464-5491.2011.03329.x. 
21 Dzida G, Karnieli E, Svendsen AL, Sølje KS, Hermanns N. SOLVE Study Group. Depressive symptoms prior to and following insulin initiation in patients with type 2 diabetes mellitus: Prevalence, risk factors and effect on physician resource utilisation. Prim Care Diabetes 2015; 9:346-53. doi:10.1016/j.pcd.2015.01.002.

22 Furler JS, Young D, Best J, et al. Can primary care team-based transition to insulin improve outcomes in adults with type 2 diabetes: the stepping up to insulin cluster randomized controlled trial protocol. Implement Sci 2014:9:20. doi:10.1186/1748-5908-9-20.

23 Schulz KF, Altman DG, Moher D. CONSORT Group. CONSORT 2010 statement: updated guidelines for reporting parallel group randomised trials. BMJ 2010;340:c332. doi:10.1136/bmj.c332.

24 Giraudeau B, Ravaud P. Preventing bias in cluster randomised trials. PLoS Med 2009;6:e1000065. doi:10.1371/journal.pmed.1000065.

25 Blackberry ID, Furler JS, Best JD, et al. Effectiveness of general practice based, practice nurse led telephone coaching on glycaemic control of type 2 diabetes: the Patient Engagement and Coaching for Health (PEACH) pragmatic cluster randomised controlled trial. BMj 2013;347:f5272. doi:10.1136/bmj.f5272.

26 Polonsky WH, Fisher L, Schikman CH, et al. Structured self-monitoring of blood glucose significantly reduces A1C levels in poorly controlled noninsulin-treated type 2 diabetes: results from the Structured Testing Program study. Diabetes Care 2011;34:262-7. doi:10.2337/ dc10-1732.

27 The Royal Australian College of General Practitioners and Diabetes Australia. General practice management of type 2 diabetes - 2014-15. Melbourne, 2014

28 Kroenke K, Spitzer RL, Williams JBW. The PHQ-9: validity of a brief depression severity measure. J Gen Intern Med 2001;16:606-13. doi:10.1046/j.1525-1497.2001.016009606.x.

29 Welch GW, Jacobson AM, Polonsky WH. The Problem Areas in Diabetes Scale. An evaluation of its clinical utility. Diabetes Care 1997;20:7606. doi:10.2337/diacare.20.5.760.
30 Richardson J, lezzi A, Khan MA, Maxwell A. Validity and reliability of the Assessment of Quality of Life (AQoL)-8D multi-attribute utility instrument. Patient 2014:7:85-96. doi:10.1007/s40271-013-0036-X.

31 The National Diabetes Service Scheme. Secondary The National Diabetes Service Scheme. https://www.ndss.com.au/the-ndss.

32 Speight J, Browne JL, Holmes-Truscott E, et al. Diabetes MILESAustralia 2011 Survey Report.Diabetes Australia, 2011.

33 Elliott L, Fidler C, Ditchfield A, Stissing T. Hypoglycemia Event Rates: A Comparison Between Real-World Data and Randomized Controlled Trial Populations in Insulin-Treated Diabetes. Diabetes Ther 2016;7:45-60. doi:10.1007/s13300-016-0157-z.

34 Frier BM, Jensen MM, Chubb BD. Hypoglycaemia in adults with insulin-treated diabetes in the UK: self-reported frequency and effects. Diabet Med 2016;33:1125-32. doi:10.1111/dme.12878.

35 Harris SB, Gerstein HC, Yale J-F, et al. Can community retail pharmacis and diabetes expert support facilitate insulin initiation by family physicians? Results of the AIM@GP randomized controlled trial. BMC Health Serv Res 2013;13:71. doi:10.1186/1472-6963-13-71.

36 Dale J, Martin S, Gadsby R. Insulin initiation in primary care for patients with type 2 diabetes: 3 -year follow-up study. Prim Care Diabetes 2010;4:85-9. doi:10.1016/j.pcd.2010.03.001.

37 Pouwer F, Hermanns N. Insulin therapy and quality of life. A review. Diabetes Metab Res Rev 2009;25(Suppl 1):S4-10. doi:10.1002/dmrr.981.

38 Gucciardi E, Espin S, Morganti A, Dorado L. Exploring interprofessional collaboration during the integration of diabetes teams into primary care. BMC Fam Pract 2016;17:12. doi:10.1186/s12875-016-0407-1.

39 O'Connor PJ, Desai JR, Butler JC, Kharbanda EO, Sperl-Hillen JM. Current status and future prospects for electronic point-of-care clinical decision support in diabetes care. Curr Diab Rep 2013;13:172-6. doi:101007/s11892-012-0350-z.

Supplementary information: supplementary appendixes 1-5 